\title{
Resolving Surface Structures of Catalytic Ru Nanoparticles during Catalysis
}

\author{
B.K. Miller and P.A. Crozier \\ School for Engineering of Matter, Transport and Energy, Arizona State University, Tempe, AZ \\ 85287-6106
}

In situ studies of catalysts in the environmental transmission electron microscope (ETEM) are of great value to elucidate the structure of a catalyst under reaction conditions. This is important, since the structure of many catalysts change at elevated temperature in the presence of reactive gases. One way to more directly relate structure to activity is to measure the activity of the catalyst while it is being observed in the TEM. This is called operando TEM. The relative catalytic activity can be measured by monitoring the amount of product gas produced by the catalytic reaction using electron energy-loss spectroscopy (EELS) and mass spectrometry [1]. This measurement is made possible through the use of a unique sample preparation, shown schematically in Figure 1.

Using an aberration-corrected ETEM, it is possible to image the surfaces of nanoparticles while they are catalysing a reaction. The basic nanoparticle structure can be modelled using a Wulff construction; for more detail, see the abstract by Walker et al. [2]. We have used an imagecorrected FEI Titan ETEM to observe Ru particles under various reaction conditions relevant to $\mathrm{CO}$ oxidation. While this reaction is simple and well-studied, the most active form of the catalyst is still debated in the literature [2]. It is agreed that the bulk of the active catalyst particles is $\mathrm{Ru}$ metal, but the surface structures which yield the highest catalytic activity are still uncertain. Furthermore, the application of this catalyst to fuel cells will depend on the preferential oxidation of small quantities of $\mathrm{CO}$ in $\mathrm{H}_{2}$ gas, also known as PROX, which has received less study.

Figure 2 shows the surface of a Ru nanoparticle at elevated temperature with several Torr of $\mathrm{H}_{2}$ gas inside the environmental cell. It is clear that such images can reveal the structures present on surface facets of the $\mathrm{Ru}$ particles. Figure 3 shows an example of the detailed information which can be obtained, in this case for a PROX experimental condition. In 2 Torr of $\mathrm{H}_{2}$ with only 0.01 Torr of $\mathrm{O}_{2}, \mathrm{RuO}_{2}$ lattice spacings are seen in a layer about $5 \AA$ thick on both the (100) and (101) surface facets, but are absent in the rest of the particle, where $\mathrm{Ru}$ metal spacings are clearly resolved. As shown in Figure 3a, these layers are absent prior to introducing the $\mathrm{O}_{2}$ indicating that they have formed due to exposure to a small amount of oxygen in a highly reducing $\mathrm{H}_{2}$ gas.

We continue to make atomic resolution observations of nanoparticle surfaces in different gas environments, coupling these observations to the measured composition of the gas inside the ETEM cell, which should reveal structure-activity relationships for this Ru catalyst.

References:

[1] B.K. Miller, P.A. Crozier Microscopy and Microanalysis 20, (2014), p.815-824.

[2] N.P. Walker, B.K. Miller, \& P.A. Crozier, ibid. (these proceedings)

[3] D. W. Goodman, C. H. F. Peden, \& M. S. Chen, Surface Science 601, (2007), p.5663-5665.

[4] The support from National Science Foundation CBET-1134464 and the use of ETEM at John M. Cowley Center for HR Microscopy at Arizona State University is gratefully acknowledged. 


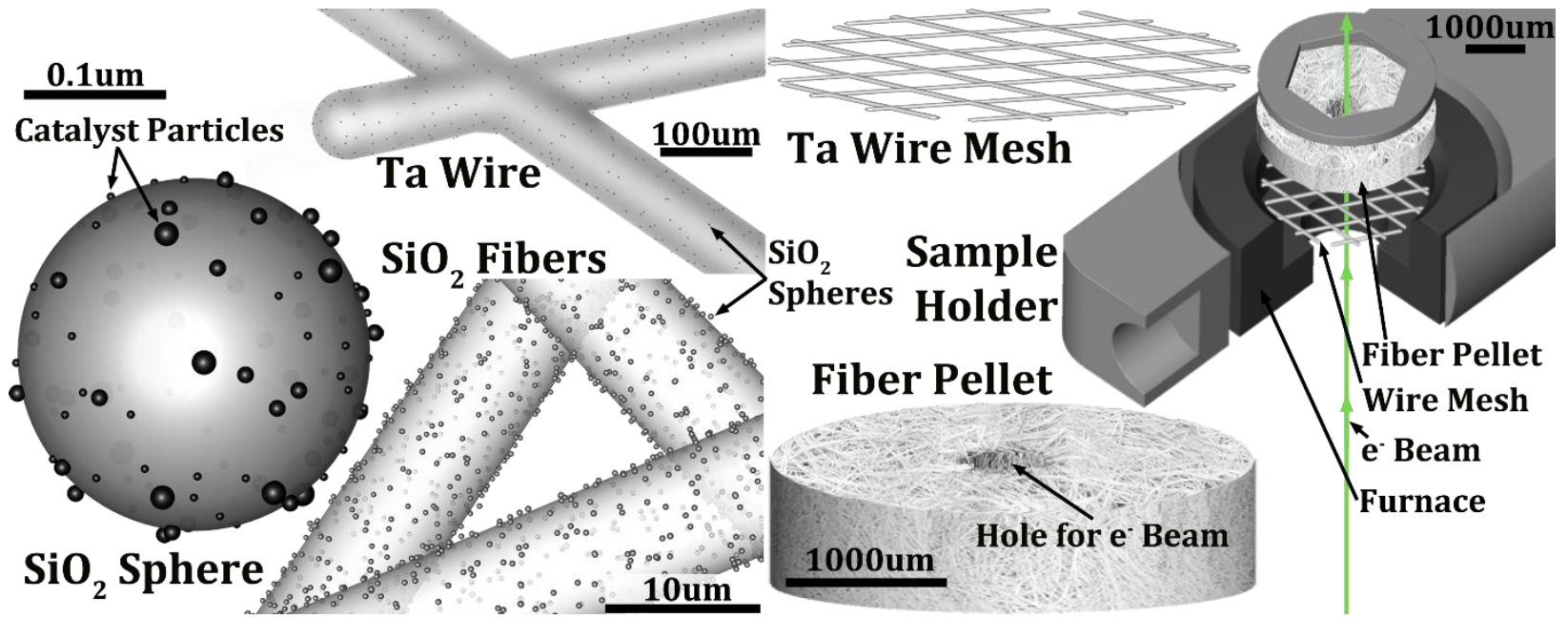

Figure 1. Schematic showing the structure of the TEM sample. a) Silica spheres of about $200 \mathrm{~nm}$ diameter are used as the catalyst support. b) The silica spheres are in turn dispersed over glass wool fibers (also silica), which have been formed into a $3 \mathrm{~mm}$ pellet. c) In the TEM, a Ta mesh grid, which also has catalyst particles dispersed over it, is placed above the pellet. Images are acquired from particles dispersed on the Ta mesh, while the entire sample contributes to the gas composition within the TEM.

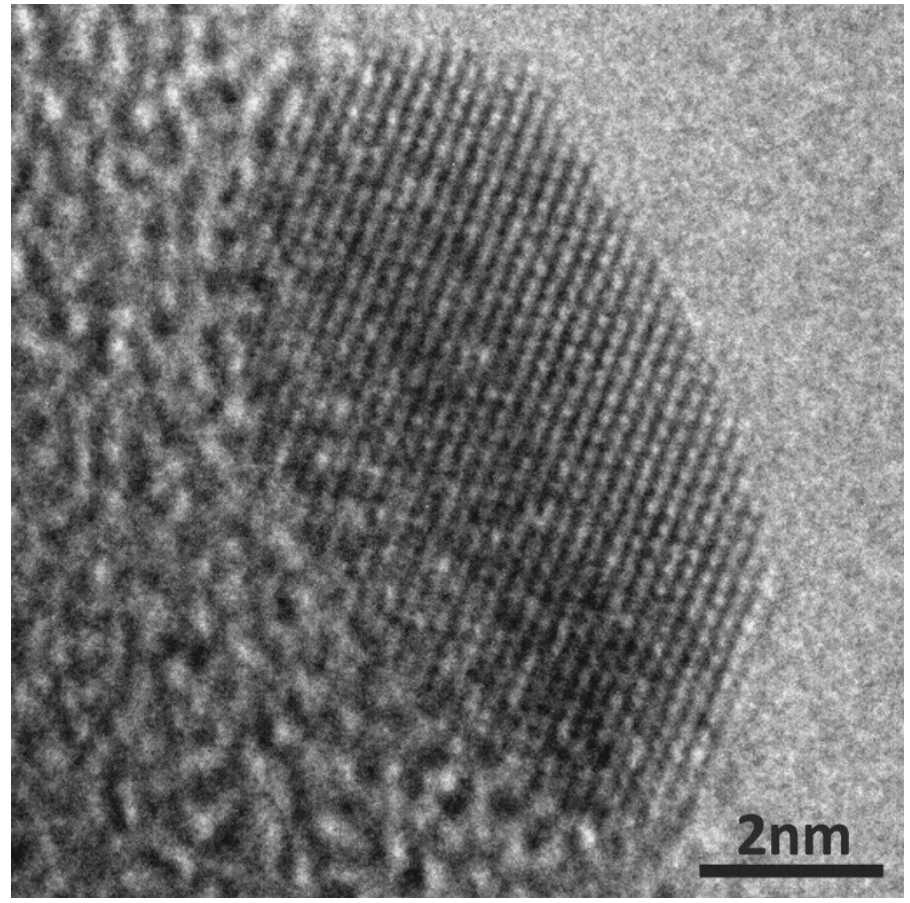

Figure 2 (above). Ru particle supported on amorphous silica. The image is a single 0.5 second acquisition with 2 Torr of $\mathrm{H}_{2}$ in the ETEM cell at $200^{\circ} \mathrm{C}$ with a TEM voltage of $80 \mathrm{kV}$. The electron dose rate is kept below $200 e / \AA^{2} \cdot s$

Figure 3 (right). Two images of the same Ru particle in different gas compositions, both at $200^{\circ} \mathrm{C}$ and $80 \mathrm{kV}$. a) 2 Torr pure $\mathrm{H}_{2}$ showing no indication of oxidation. b) 2 Torr $\mathrm{H}_{2}$ with 0.01 Torr $\mathrm{O}_{2}$, showing a $0.5 \mathrm{~nm}$ layer of $\mathrm{RuO}_{2}$ on the (100) and (101) Ru surfaces.

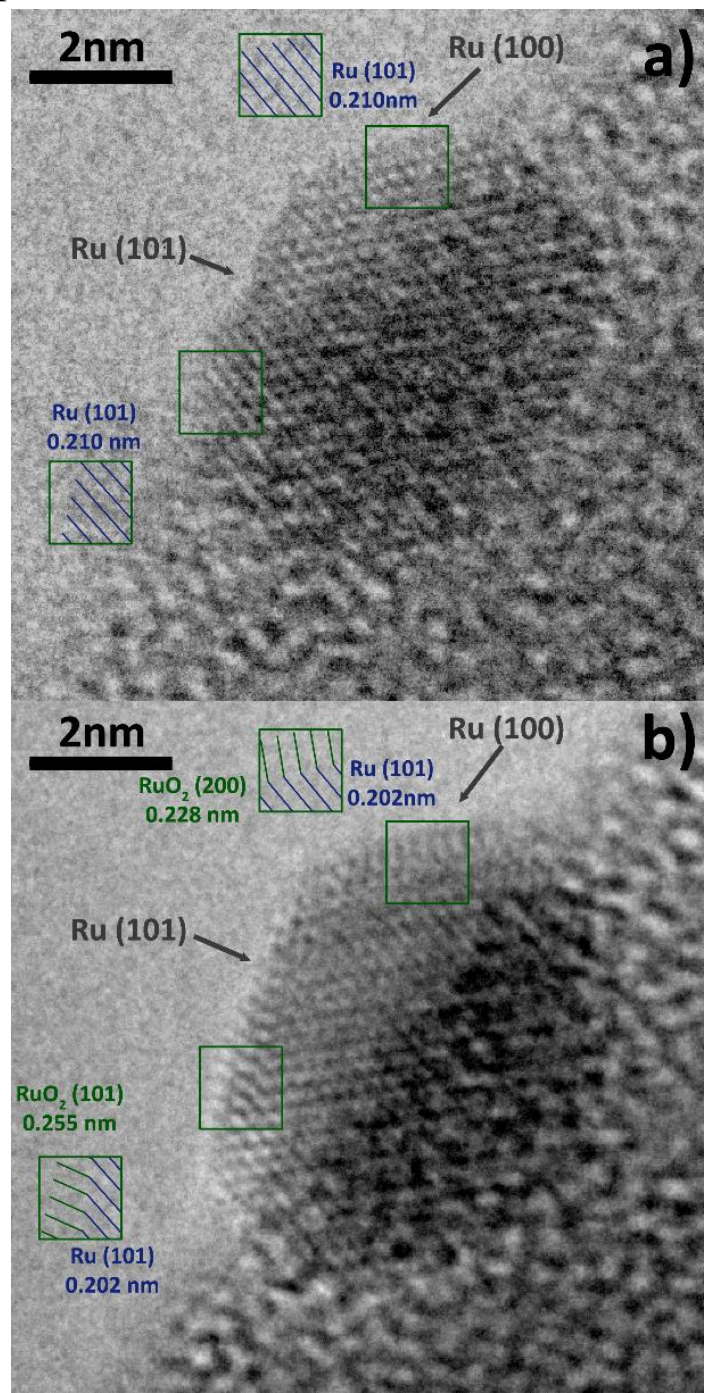

